\title{
Polymorphous presentations in vitelliform macular dystrophy: subretinal neovascularisation and central choroidal atrophy
}

\author{
KENNETH G. NOBLE, BARRY M. SCHER, AND RONALD E. CARR \\ From the Department of Ophthalmology, New York University Medical Center, New York
}

SUMmaRY Two dominantly inherited macular dystrophies demonstrate the difficulty in establishing a diagnosis based on the fundus appearance. In 1 family the propositus presented with unilateral retinal haemorrhage associated with subretinal choroidal neovascularisation which remained unilateral over an 8-year period. In the other family the propositus presented with bilateral central choroidal atrophy. All affected family members had an abnormal electro-oculogram and a normal electroretinogram, suggesting the diagnosis of vitelliform macular dystrophy. Since vitelliform macular dystrophy has a wide range of expressivity, with polymorphous appearances of the fundus, the diagnosis is best made by the presence of a dominant mode of inheritance and an abnormal electro-oculogram.

The hereditary macular dystrophies usually present with a characteristic macular lesion which is bilateral and often symmetrical in appearance. The family history, age of onset, progressive course, and absence of extramacular involvement are additional helpful requisites in making the diagnosis.

This report discusses 2 families with vitelliform macular dystrophy in which the propositus of 1 family presented with unilateral subretinal neovascularisation of the macula and the propositus of the second family presented with bilateral central choroidal atrophy.

\section{Case reports}

FAMILY 1

Four generations of this family (Fig. 1) of Germanic ancestry were studied and 5 family members were examined. All family members were in excellent general health.

Case 1 (III-2) Propositus (Figs. 2 and 3)

This 28-year-old white woman was first told of diminished vision at the age of 8 years. Her father has had poor vision in each eye since childhood. She was first seen in the Retinal Clinic at New York University Medical Center at age 20 years.

Her vision was $6 / 9(20 / 30)$ OD and 6/4.5 (20/15)

Address for reprints: Dr Kenneth G. Noble, Department of Ophthalmology, New York University Medical Center, 550 First Avenue, New York, New York 10016, USA
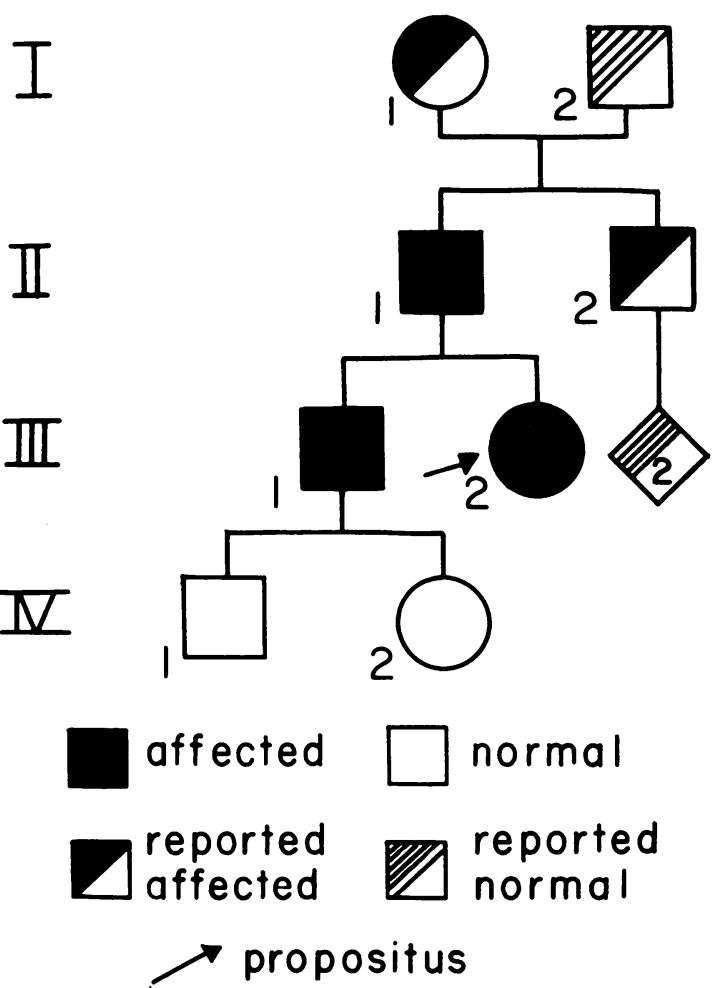

Fig. 1 Pedigree of family 1 with vitelliform macular dystrophy. Patients $I V-I$ and $I V-2$ did not have an electro-oculogram 


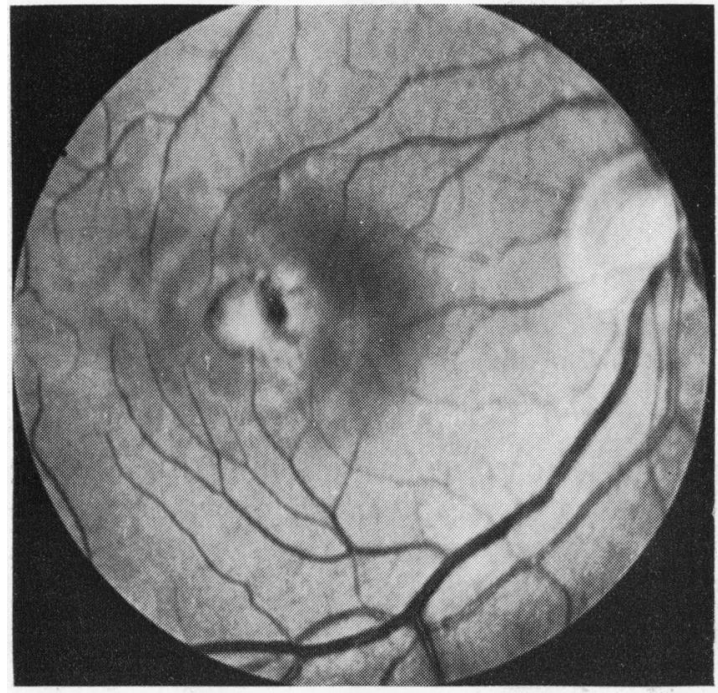

$2 a$

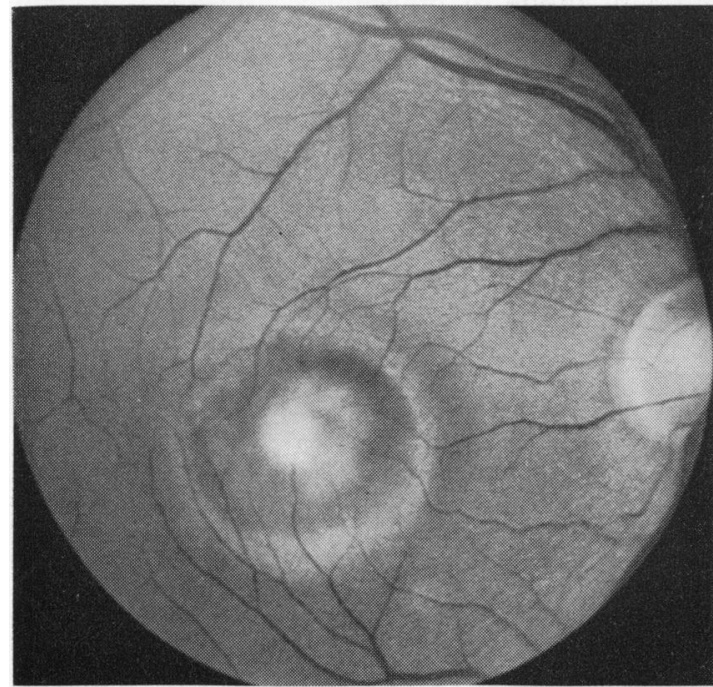

$2 c$

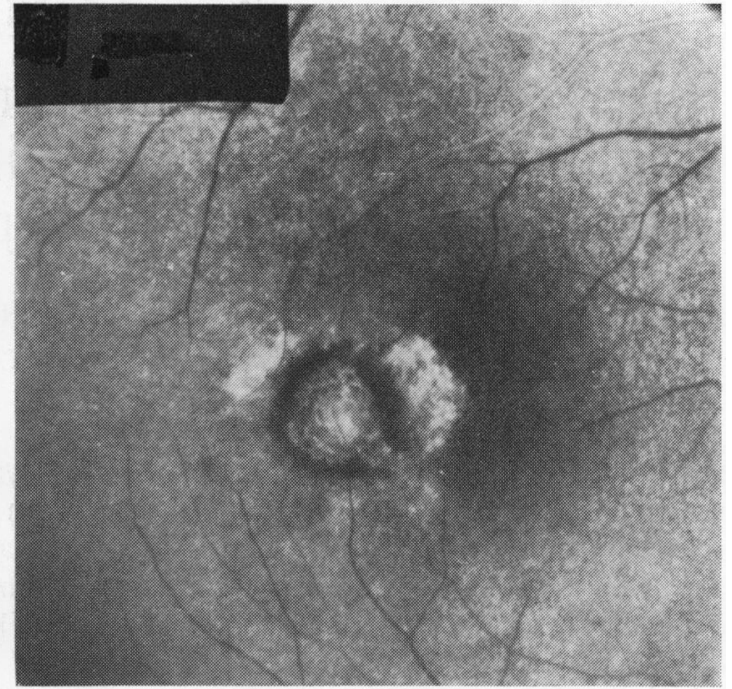

$2 b$

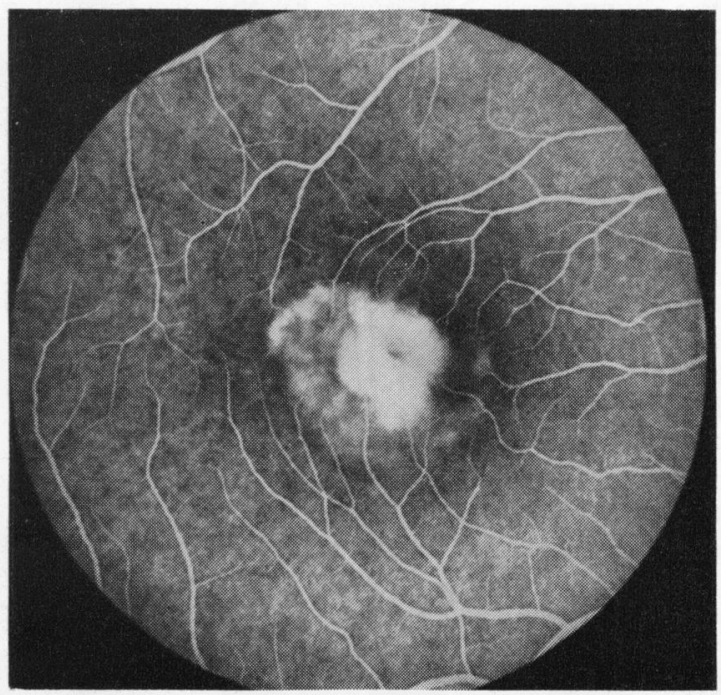

$2 \mathrm{~d}$

Fig. 2 Case 1, right eye. At the initial examination a greenish grey membrane is present just temporal to the fovea surrounded by pigment and haemorrhage (Fig. 2a). Fluorescein angiography shows a subretinal neovascular network adjacent to the fovea (Fig. 2b). Examination 8 years later shows replacement of the neovascular network by a gliotic mound (Fig. 2c), and the angiogram shows staining of the glial tissue and surrounding transmission hyperfluorescence (Fig. 2d)

OS, with a mild hyperopia. The macula of the right eye showed a greenish grey membrane just temporal to the fovea, which was surrounded by pigmentation temporally and by subretinal haemorrhage at the nasal border. The macula of the left eye showed only a few small drusen. The optic disc, retinal vessels, and peripheral retina were normal in each eye.

Fluorescein angiography demonstrated in the right eye a subretinal network of vessels abutting on the temporal fovea. These vessels filled in the choroidal phase and subsequently leaked dye into the subretinal space. The left eye showed a transmitted hyperfluorescence in the macula.

In view of the history of her father's poor vision since childhood a tentative diagnosis of pseudoinflammatory macular dystrophy was made. 


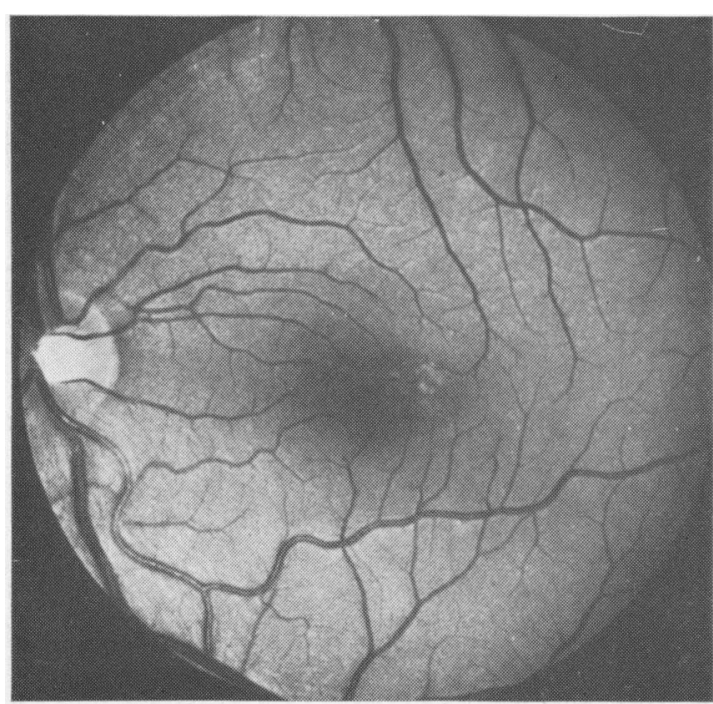

$3 a$

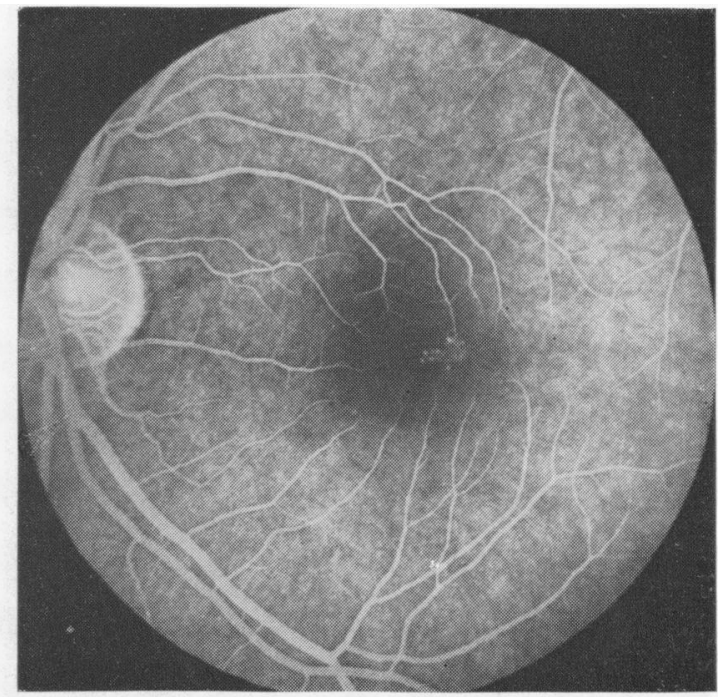

$3 \mathrm{~b}$

Fig. 3 Case 1, left eye. The most recent examination shows a few drusen temporal to the fovea (Fig. $3 a$ ), which hyperfluoresce on fluorescein angiography (Fig. $3 b$ ). This picture has remained unchanged over an 8-year period

The patient was seen for follow-up examinations over the next 2 years without a change in vision or fundus appearance. She was then lost to follow-up until her most recent examination at age 28 years. The visual acuity was $6 / 6(20 / 20)$ in each eye. The neovascular tissue had been replaced by a gliotic mound, which was surrounded by mild pigmentary loss. The left eye was unchanged. Fluorescein angiography of the right eye demonstrated staining of the gliotic tissue surrounded by transmitted hyperfluorescence.

The electro-oculogram was abnormal in each eye (OD $138 \%$; OS $129 \%$ Arden index), and the electroretinogram was normal.

\section{Case 2 (II-2) (Fig. 4)}

The father of the propositus, first seen at age 60 , gave a history of progressive visual loss in each eye since age 6 years which stabilised at age 50 years. He reported that his mother and brother had similar visual difficulties.

His best corrected vision was $6 / 60(20 / 200)$ OU with correction $\left(+7 \cdot 75-3.00 \times 100^{\circ} \mathrm{OD}\right.$ and $+7 \cdot 00$ $-0.75 \times 85^{\circ} \mathrm{OS}$ ). The macula of each eye showed pigment atrophy with some glial tissue proliferation and a scattering of pigment. Fluorescein angiography demonstrated transmitted hyperfluorescence and staining in the affected maculae.

Re-examination 7 years later revealed no change in vision or fundus appearance. Electro-oculography of each eye was abnormal (OD 167\%; OS $161 \%$
Arden index), and the electroretinogram was normal.

Case 3 (III-1) (Fig. 5)

This 30-year-old man, the brother of the propositus, denied any visual symptoms. The uncorrected visual acuity was $6 / 4.5(20 / 15)$ OU. In the macula of each eye there was a symmetrical circular loss of pigment $(0.5 \mathrm{~mm}$ in size) located just superotemporal to the normal foveal reflex. This area demonstrated a transmitted hyperfluorescence on fluorescein angiography. Electro-oculography was abnormal (OD 126\%; OS 147\% Arden index), and the electroretinogram was normal.

His 2 young children, age 4 (IV-1) and 1 (IV-2), had normal fundus examinations. Electro-oculography could not be performed at that time.

\section{Comment}

This family exhibited the wide range of expressivity often seen in a dominant macular dystrophy. The propositus presented with unilateral subretinal neovascularisation associated with a mild, nonprogressive visual loss noted during childhood. After an 8-year follow-up the neovascular tissue was replaced by glial tissue, with improvement of vision to $6 / 6(20 / 20)$.

The father had a history of slowly progressive visual loss since childhood, a visual acuity of $6 / 60$ (20/200), and a bilateral, symmetrical macular 


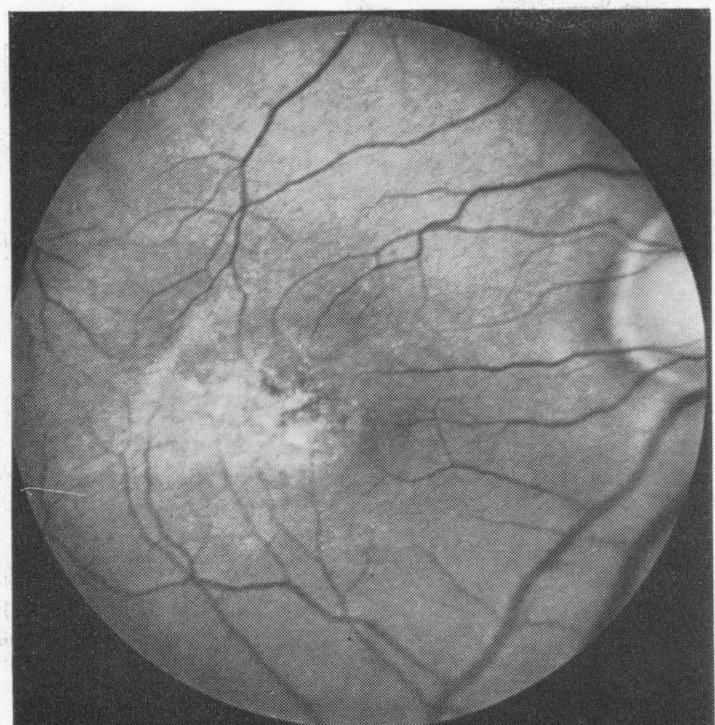

$4 a$

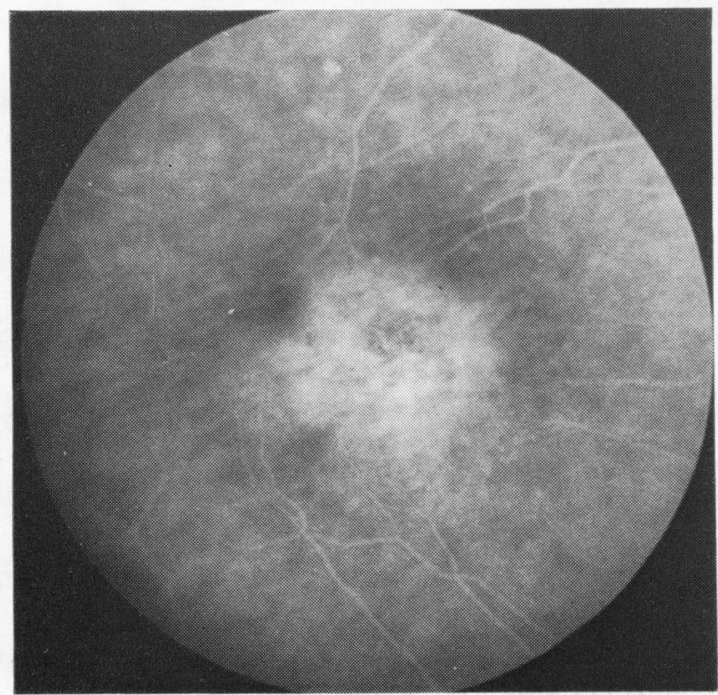

$4 c$

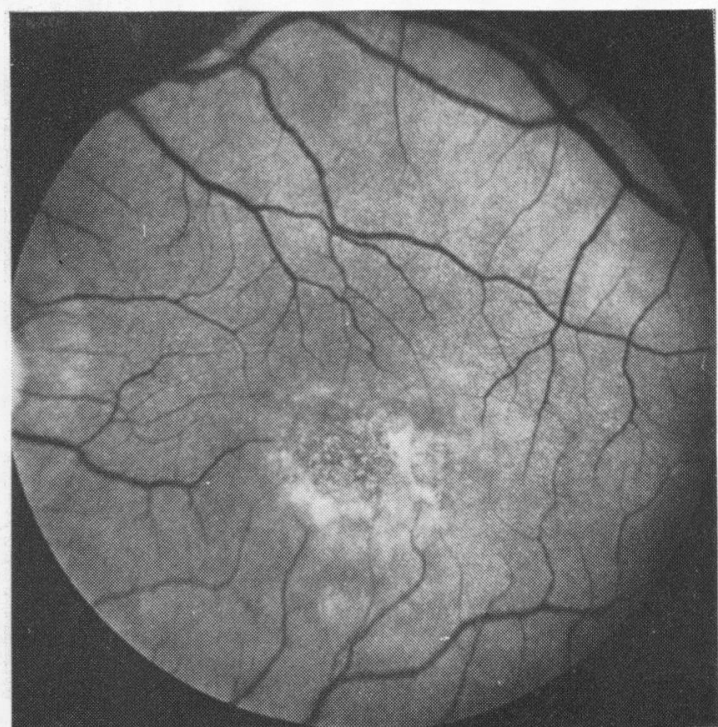

$4 b$

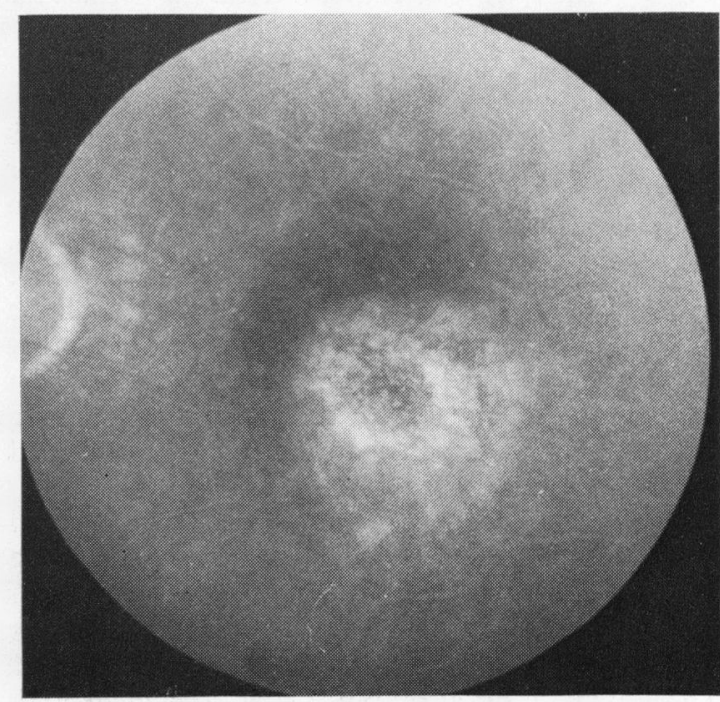

4d

Fig. 4 Case 2. Both eyes have a symmetrical loss of pigment in the macula associated with some glial tissue proliferation and pigment clumping (Figs. $4 a$ and $b$ ). Fluorescein angiography (Figs. $4 c$ and d) demonstrates a transmitted hyperfluorescence

degeneration consisting of pigment atrophy, pigment accumulation, and gliosis.

The brother of the propositus was asymptomatic with a vision of $6 / 4.5(20 / 15) \mathrm{OU}$, but his maculae showed a bilateral, symmetrical loss of pigment just adjacent to the fovea.

All family members had an abnormal electrooculogram and a normal electroretinogram.
FAMILY 2

Three generations of this white family (Fig. 6) were studied and 7 family members were examined. All family members were in excellent general health.

Case 4 (II-I) Propositus (Figs. 7 and 8)

This 57-year-old man had a history of slowly progressive visual loss in each eye since age 35 . His 


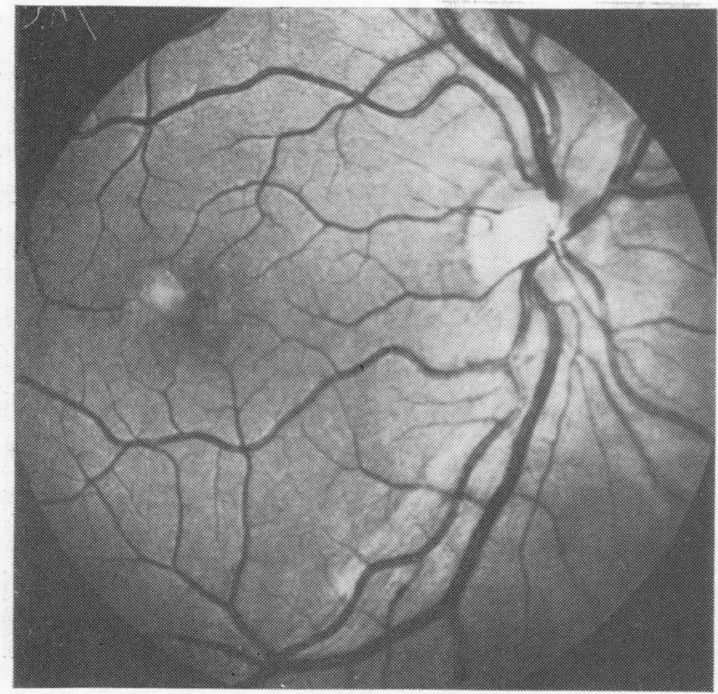

$5 \mathrm{a}$

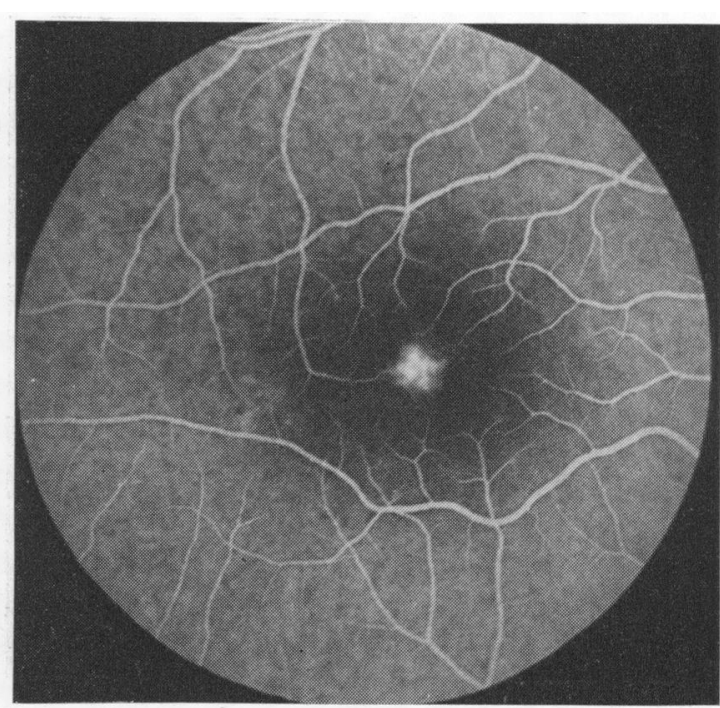

$5 b$

Fig. 5 Case 3. Adjacent to the fovea is a circular loss of pigment (Fig. 5a) which hyperfluoresces on angiography (Fig. 5b)

mother had poor vision of unknown aetiology which began in the fourth decade.

His visual acuity was $6 / 60(20 / 200)$ OU. The macula of each eye showed a well demarcated loss of the pigment epithelium and the choroidal vessels were clearly visible. Superior to these areas were clear detachments of the pigment epithelium in each eye. The posterior pole of each eye showed multiple irregular atrophic areas similar to drusen. Visual fields showed small central scotoma and full peripheral fields. The Farnsworth Panel D-15 test demonstrated non-specific colour confusion in each eye.

The diagnosis at this time was central areolar choroidal dystrophy.

Follow-up examination was performed 8 years later. The visual acuity remained at $6 / 60(20 / 200)$

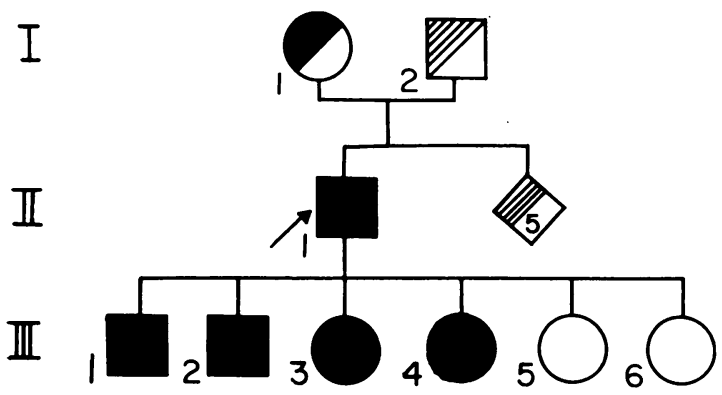

Fig. 6 Pedigree of family 2 with vitelliform macular dystrophy (see Fig. 1 for key)
OU. The visual field was similarly unchanged. In the right eye the pigment epithelial detachment superior to the macula had flattened and showed a picture of choroidal atrophy. A new pigment epithelial detachment was seen between this area and the disc. In the left eye the pigment epithelial detachment superior to the macula had likewise flattened, resulting in an ovoid area of choroidal atrophy. A new large pigment epithelial detachment was located temporal to the macula. The area of choroidal atrophy in the macula had enlarged in each eye.

Fluorescein angiography showed a mild loss of the choriocapillaris in the areas showing choroidal atrophy, and the pigment epithelial detachments showed hypofluorescence. Throughout the posterior pole were many small, dot-like areas of hyperfluorescence.

The electro-oculogram was abnormal $(100 \% \mathrm{OU}$ Arden index) and the electroretinogram was normal.

Examination 2 years later showed that the previously noted pigment epithelial detachments had flattened in each eye. In the left eye this resulted in choroidal atrophy. No new pigment epithelial detachments were seen.

Case 5 (III-1) (Fig. 9a)

The asymptomatic son of Case 4 was seen at age 29 . His visual acuity was $6 / 7 \cdot 5+(20 / 25+)$ OU. The macula of each eye showed a clear cystic lesion within which were scattered yellowish white material. 


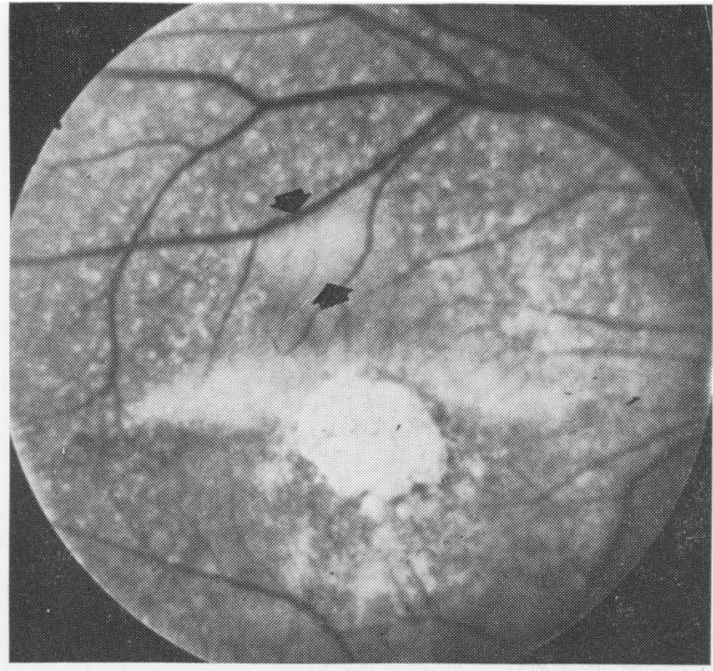

$7 a$

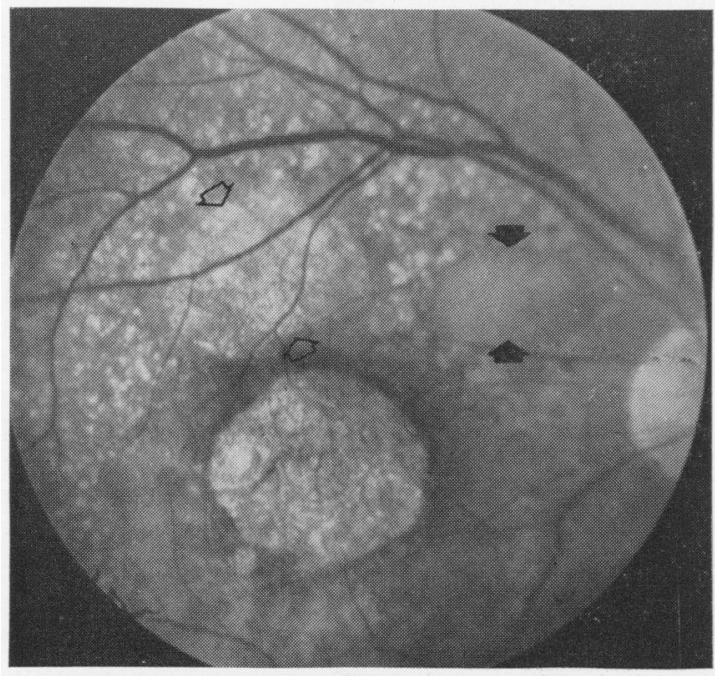

$7 c$

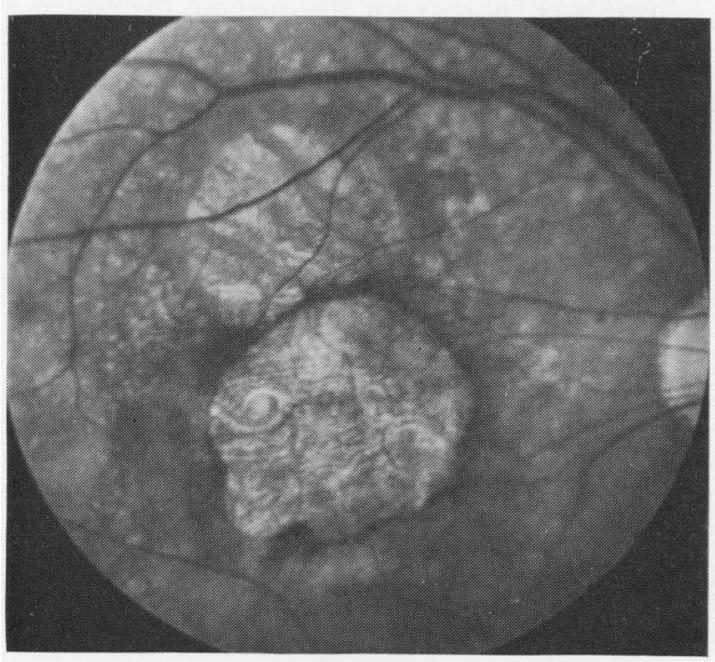

7 e

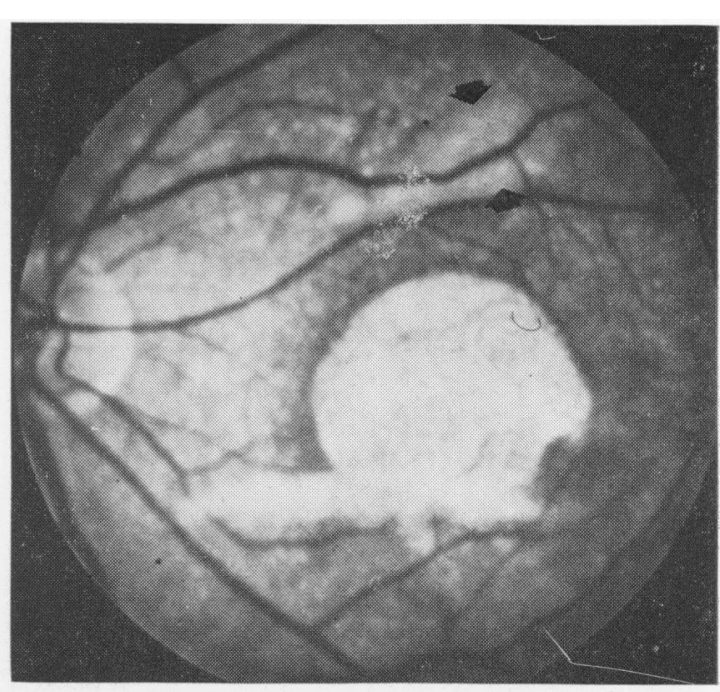

$7 b$

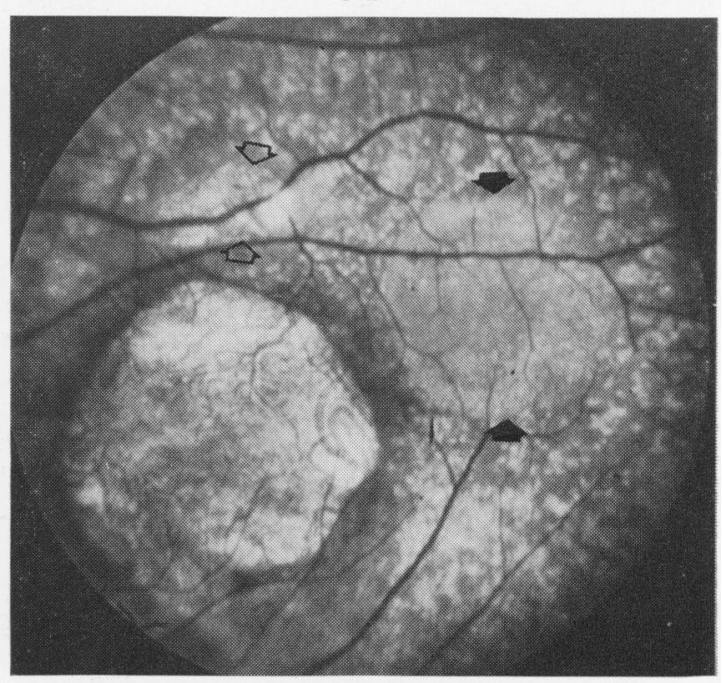

\section{$7 d$}

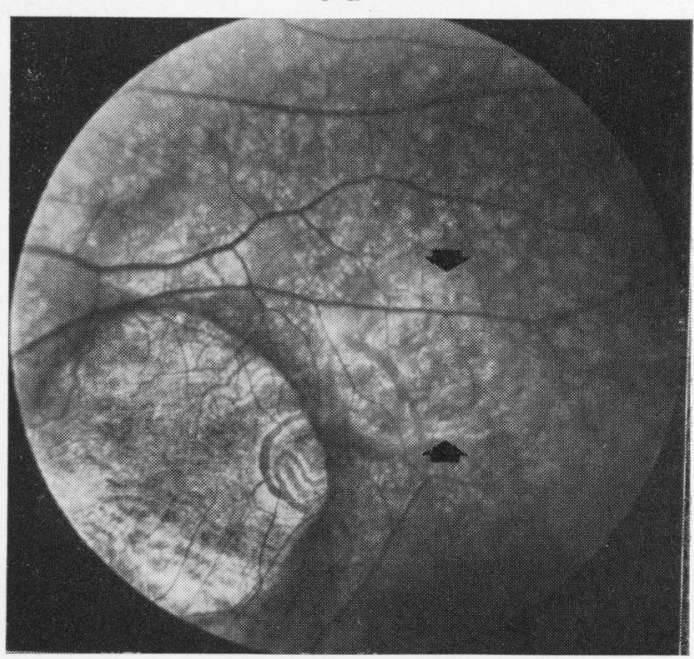

$7 f$

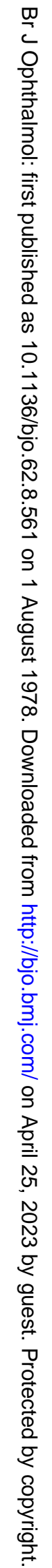


Fluorescein angiography demonstrated areas of hypc- and hyperfluorescence in the affected maculae. The electro-oculogram was abnormal $(100 \%$ OU Arden index) and the electroretinogram was normal.

Case 6 (III-2) (Fig. 9b)

This 27-year-old man was asymptomatic, with a visual acuity of $6 / 6(20 / 20)$ OU. The macula of each eye had a mild loss of pigment surrounded by a few yellowish white flecks. The hypo- and hyperfluorescence on angiography reflected the pigment alterations. The electro-oculogram was abnormal ( $100 \%$ OU Arden index), and the electroretinogram was normal.

\section{Case 7 (III-3) (Fig. 9c)}

The asymptomatic 13-year-old daughter of Case 4 was examined as part of a family study. Visual acuity was $6 / 12(20 / 40)$ OD and 6/6 (20/20) OS. The macula of each eye showed small yellowish white gliotic tissue with surrounding pigment atrophy. Visual fields were normal. Colour vision testing with the AOHRR plates and the Farnsworth Panel D-15 was normal.

Follow-up examination 8 years later revealed a decrease in vision in the right eye from $6 / 12(20 / 40)$ to $6 / 60(20 / 200)$, while the left eye remained at $6 / 6$ $(20 / 20)$. A right exotropia was evident. There was a slight increase in the amount of gliosis and pigment atrophy of the maculae, but the appearance was essentially unchanged. The maculae had a symmetrical appearance despite the markedly disparate visual acuities. Fluorescein angiography in each eye showed hyper- and hypofluorescence associated with the pigmentary alterations. There was no evidence of choroidal atrophy or dye leakage. The electro-oculogram was abnormal $(100 \%$ OU Arden index), and the electroretinogram was normal.

\section{Case 8 (III-4) (Fig. 9d)}

This 9-year-old daughter of Case 4 was asymptomatic with a vision of $6 / 15(20 / 50)$ OD and 6/6 $(20 / 20)$ OS. The macula of each eye showed a clear oval cystic area 1 to $1 \frac{1}{2}$ disc diameter in size. Within the cysts were scattered yellowish white gliotic material. Colour vision testing on the AOHRR

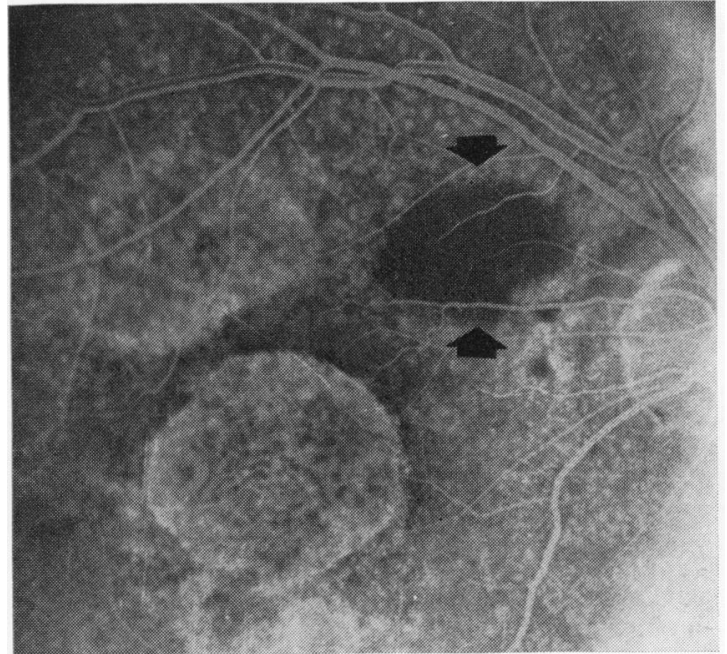

$8 a$

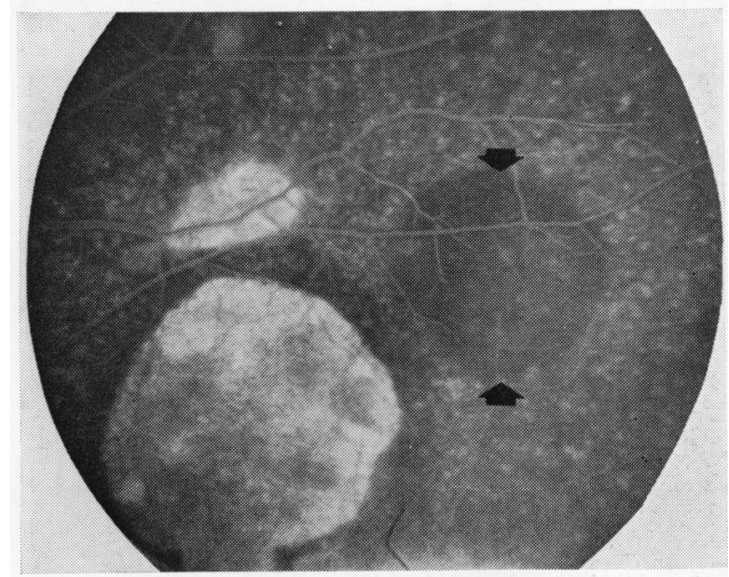

$8 \mathrm{~b}$

Fig. 8 Case 4. Fluorescein angiography performed at the 8-year follow-up examination shows a mild loss of the choriocapillaris in the areas of choroidal atrophy (Fig. 8a) with staining and transmitted hyperfluorescence in the late stages (Fig. 8b). The pigment epithelial detachments block the dye transmission resulting in hyperfluorescence (closed arrows). The speckled dots of hyperfluorescence correspond in most instances to the atrophic areas seen on ophthalmoscopy

Fig. 7 Case 4. At the time of initial examination (Figs. 7a and b) the macula of each eye showed a well circumscribed circular loss of pigment with the choroidal vessels easily seen. Superior to the macula in each eye is a clear detachment of the pigment epithelium (closed arrows). Throughout the posterior pole of each eye are small round atrophic areas similar to drusen. Eight years later (Figs. $7 c$ and d) the areas of central choroidal atrophy have enlarged. The previous pigment epithelial detachments have flattened and now show early evidence of pigmentary and choroidal atrophy (open arrows). A newly-formed detachment is seen in each eye, in the right eye adjacent to the superior aspect of the disc (Fig. 7c) and in the left eye temporal to the macula (Fig. 7d) (closed arrows). At the most recent examination the detachments have flattened in each eye (Fig. 7e). In the left eye (Fig. 7f) this has led to choroidal atrophy. The areas of choroidal atrophy in each eye continue to enlarge 


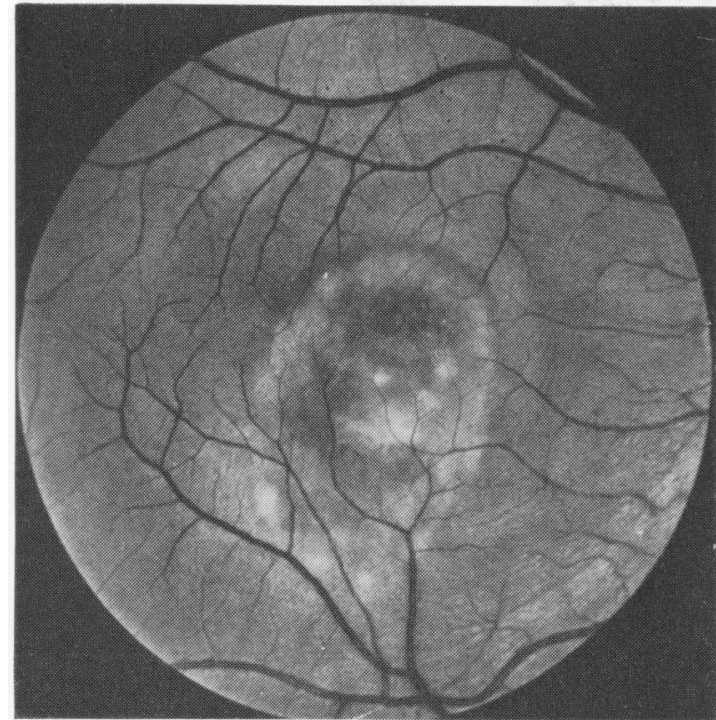

$9 a$

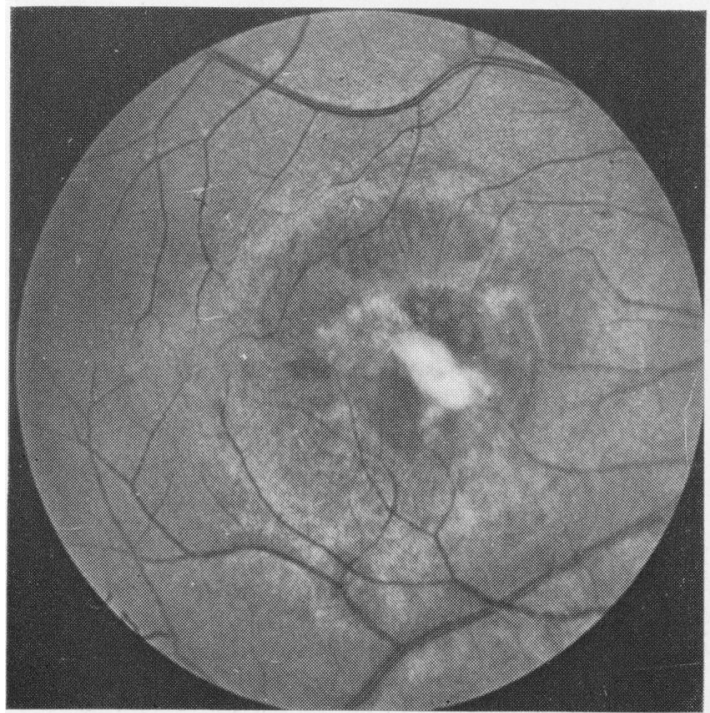

9c

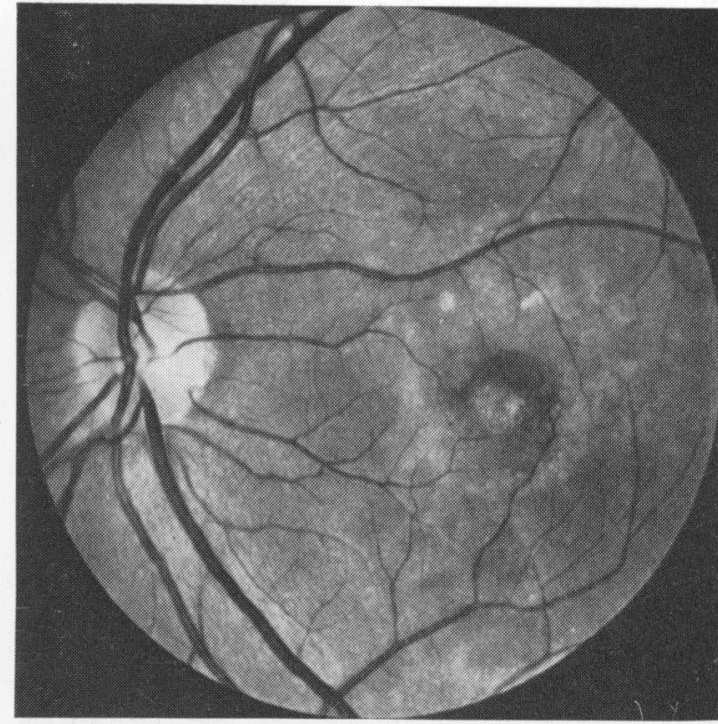

$9 b$

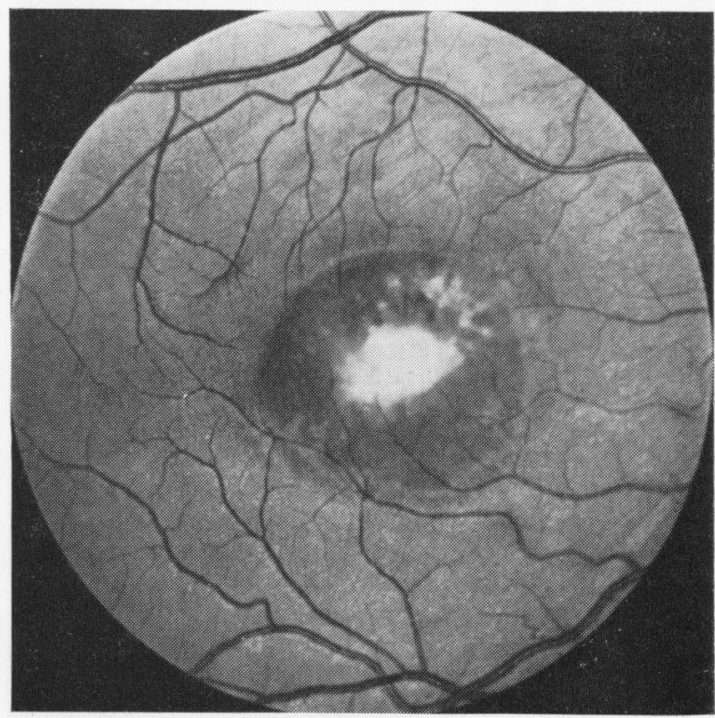

9d

Fig. 9 Representative photographs of the offspring of Case 4. The appearance of the macula in these 4 individuals included flattened cysts (Case 5, Fig. 9a), pigment atrophy (Case 6, Fig. 9b), and gliosis (Cases 7 and 8. Figs. $9 \mathrm{c}$ and d)

plates was normal. The visual field was normal in each eye.

Follow-up examination 8 years later showed a slight decrease in vision in the right eye to $6 / 24$ $(20 / 80)$, while the left eye remained at $6 / 6(20 / 20)$. The fundus appearance was unchanged. Fluorescein angiography demonstrated hypo- and hyperfluorescence associated with pigment alterations, and there was no choroidal atrophy or dye leakage. The electro-oculogram was abnormal $(100 \%$ OU Arden index).

Examination 2 years later showed essentially no change in the visual acuity or fundus appearance.

Case 9 (III-5)

This woman was seen at age 20 and denied any 
ocular symptoms. The vision was $6 / 6(20 / 20) \mathrm{OU}$, the fundus examination was normal, and the electrooculogram was normal ( $230 \%$ OU Arden index).

Case 10 (III-6)

This woman was seen at age 6 and 15 . On both visits the vision was $6 / 6(20 / 20)$ OU and the fundus examination was normal. The electro-oculogram performed at the last visit was normal $(230 \%$ OU Arden index).

\section{Comment}

The propositus in this family with an autosomal dominant macular dystrophy presented with bilateral visual loss (6/60) associated with choroidal atrophy in the macula of each eye typical of central areolar choroidal dystrophy.

The 4 affected children all had bilateral symmetrical macular changes which differed from those of their father. The fundus appearance consisted of clear cysts filled with yellowish white material, gliosis, or mild pigmentary loss. All children were asymptomatic, with vision in the better eye of $6 / 6$ $(20 / 20)$.

The electro-oculogram was abnormal and the electroretinogram was normal in all family members who were affected.

The progression of fundus lesions in the propositus suggests the following sequence of events: first, there occurs a clear detachment of the pigment epithelium, which contains material that does not permit fluorescence of dye (resulting in hypofluorescence); these detachments flatten, resulting in atrophy of the pigment epithelium and the choriocapillaris. This is an active process, as evidence by the appearance of new pigment epithelial detachments in each eye of this 65 -year-old man.

\section{Discussion}

The association of an autosomal dominant macular dystrophy with an abnormal electro-oculogram and a normal electroretinogram strongly suggests a diagnosis of vitelliform macular dystrophy. Vitelliform macular dystrophy need not always show the intact or ruptured cyst stage thought to be characteristic of this disease. Pigment atrophy, drusen, subretinal haemorrhages (Braley, 1966; Benson et al., 1975), choroidal neovascularisation (Miller et al., 1976), gliosis, and choroidal atrophy (Deutman, 1971) may all be seen.

Similarly, vitelliform dystrophy may be unilateral (François et al., 1967; Deutman, 1969) or so asymmetrical as to appear unilateral, so that a diagnosis of an inherited macular dystrophy is not considered. When the unilateral fundus presentation is atypical, as in Case 1, the diagnosis may be extremely difficult to make.

The differential diagnosis of unilateral subretinal neovascularisation in children includes the presumed ocular histoplasmosis syndrome, inflammations, trauma, and an idiopathic variety (Braunstein et al., 1974; Cleasby, 1976). Hereditary ocular diseases, which may show unilateral subretinal neovascularisation, are angioid streaks (Gass, 1977, p. 78), dominant drusen of Bruch's membrane, optic nerve drusen (Friedman et al., 1977), and myopia (Beyrer, 1977). Pseudoinflammatory macular dystrophy exhibits subretinal neovascularisation as the earliest finding, but the onset is usually in the 4th decade with bilateral symmetrical macular involvement (Sorsby et al., 1949; Carr et al., in press). A dominantly inherited macular dystrophy associated with aminoaciduria has been described in one family (Lefler et al., 1971), and a few family members have had subretinal fibrosis suggesting previous subretinal neovascularisation.

Although uncommon, bilateral symmetrical choroidal atrophy of the macula has been seen in individuals with vitelliform macular dystrophy (Deutman, 1971). The fundus and angiographic appearance may be identical to that seen in central areolar choroidal dystrophy. Since both these hereditary macular dystrophies are inherited in an autosomal dominant manner (Sandvig, 1955; Carr, 1965), the differential is best made by the abnormal electro-oculogram in vitelliform macular dystrophy. The differential diagnosis of bilateral central choroidal atrophy has been previously discussed (Noble, 1977) and includes Stargardt's disease (Irvine and Wergeland, 1972), dominant progressive cone dystrophy (Krill and Deutman, 1972), acquired macular degenerations (geographic atrophy of the retinal pigment epithelium) (Blair, 1975), geographic (Schatz et al., 1974), serpiginous (Gass, 1977 , p. 112) choroidopathy, and chorioretinal inflammations (macular 'coloboma').

In view of the polymorphous fundus appearance in vitelliform macular dystrophy the electro-oculogram is of prime importance in establishing the diagnosis, since it is abnormal in all affected family members (Deutman, 1959; Cross and Bard, 1974). In none of the other disorders discussed in the differential diagnosis is the electro-oculogram uniformly abnormal.

We wish to thank Dr Samuel Sokol who performed the electro-oculogram on Case 7.

Supported in part by grant EY00213 from the National Eye Institute, National Institutes of Health, Bethesda, Maryland, USA 


\section{References}

Benson, W. E., Kolker, A. E., Enoch, J. M., Van Loo, J. A., and Honda, Y. (1975). Best's vitelliform macular dystrophy. American Journal of Ophthalmology, 79, 59-66.

Beyrer, C. R. (1977). Subretinal neovascularization in high myopia. Presented at the AMA Annual Convention, San Francisco.

Blair, C. J. (1975). Geographic atrophy of the retinal pigment epithelium. Archives of Ophthalmology, 93, 19-25.

Braley, A. E. (1966). Dystrophy of the macula. American Journal of Ophthalmology, 61, 1-24.

Braunstein, R. A., Rosen, D. A., and Bird, A. C. (1974). Ocular histoplasmosis syndrome in the United Kingdom. British Journal of Ophthalmology, 58, 893-898.

Carr, R. E. (1965). Central areolar choroidal dystrophy. Archives of Ophthalmology, 73, 32-35.

Carr, R. E., Noble, K. G., and Nasaduke, I. (1978). Pseudoinflammatory macular dystrophy. Transactions of the American Ophthalmological Society, 75, 255-271.

Cleasby, G. W. (1976). Idiopathic focal subretinal neovascularisation. American Journal of Ophthalmology, 81, 590-596.

Cross, H. E., and Bard, L. (1974). Electro-oculography in Best's macular dystrophy. American Journal of Ophthalmology, 77, 46-50.

Deutman, A. F. (1969). Electro-oculography in families with vitelliform dystrophy of the fovea. Archives of Ophthalmology, 81, 305-316.

Deutman, A. F. (1971). The Hereditary Dystrophies of the Posterior Pole, p. 230. Van Gorcum: Assen.

Francois, J., DeRouck, A., and Fernandez-Sasso, D. (1967). Electro-oculography in vitelliform degeneration of the macula. Archives of Ophthalmology, 77, 726-733.

Friedman, A. H., Beckerman, B., Gold, D. H., Walsh, J. B., and Gartner, S. (1977). Drusen of the optic disc. Survey of Ophthalmology, 21, 375-390.

Gass, J. D. M. (1977). Stereoscopic Atlas of Macular Diseases, 2nd edn. C. V. Mosby: St. Louis.

Irvine, A. R., and Wergeland, F. L., Jr. (1972). Stargardt's hereditary progressive macular degeneration. British Journal of Ophthalmology, 56, 817-826.

Krill, A. E., and Deutman, A. F. (1972). Dominant macular degenerations: the cone dystrophies. American Journal of Ophthalmology, 73, 352-369.

Lefler, W. H., Wadsworth, J. A. C., and Sidbury, J. B. (1971). Hereditary macular degeneration with aminoaciduria. American Journal of Ophthalmology, 71, 224 230.

Miller, S. A., Bresnick, G. H., and Chandra, S. R. (1976). Choroidal neovascular membrane in Best's vitelliform macular dystrophy. American Journal of Ophthalmology, 82, 252-255.

Noble, K. G. (1977). Central areolar choroidal dystrophy. American Journal of Ophthalmology, 84, 310-318.

Sandvig, K. (1955). Familial, central, areolar, choroidal atrophy of autosomal dominant inheritance. Acta Ophthalmologica, 33, 71-78.

Schatz, H., Maumenee, A. E., and Patz, A. (1974). Geographic helicoid peripapillary choroidopathy: clinical presentation and fluorescein angiographic findings. Transactions of the American Academy of Ophthalmology and Otolaryngology, 78, 747-761.

Sorsby, A., Joll-Mason, M. E., and Gardener, N. (1949). A fundus dystrophy with unusual features. British Journal of Ophthalmology, 33, 67-97. 